\title{
When is quality assurance a constructive force in engineering education?
}

\author{
PEARS, A., DANIELS, M., NYLEN, A., and MCDERMOTT, R.
}

2019

(C) 2020 IEEE. Personal use of this material is permitted. Permission from IEEE must be obtained for all other uses, in any current or future media, including reprinting/republishing this material for advertising or promotional purposes, creating new collective works, for resale or redistribution to servers or lists, or reuse of any copyrighted component of this work in other works. 


\title{
When is Quality Assurance a Constructive Force in Engineering Education?
}

\author{
Arnold Pears*, Mats Daniels ${ }^{\dagger}$, Aletta Nylén ${ }^{\dagger}$, Roger McDermott ${ }^{\ddagger}$ \\ * Department of Learning in Engineering Sciences, KTH Royal Institute of Technology, Sweden \\ Email: pears@kth.se \\ ${ }^{\dagger}$ Department of Information Technology Uppsala University, Uppsala, Sweden \\ Email: firstname.lastname@it.uu.se \\ $\S$ Robert Gordon University, Aberdeen, Scotland \\ Email: roger.mcdermott@rgu.ac.uk
}

\begin{abstract}
Quality assurance processes in education have been a key area of engineering education development for several decades. ABET, ENQA - The European Association for Quality Assurance in Higher Education, as well as other agencies in Europe and the Asia Pacific have largely converged on a set of high level graduate outcomes, widely considered to be those most relevant to the engineering professions. We suggest that outcome assessment can be classified into four major approaches, education as a service (with the focus on identifying customers), as a process (with a focus on describing the formative impact of curriculum on learners), as a Body of Knowledge (with a focus on transmission of that knowledge and the generation of artefacts), and finally as expansion of the individual (focusing on holistic development of the intellect).
\end{abstract}

\section{INTRODUCTION}

This paper revisits the issues surrounding quality assurance processes drawing a comparison of national and institutional regulatory structures, quality key performance indicators, legislative structures and academic perspectives from three European countries. The authors leverage their extensive experience as deans of education, department heads, and pedagogical curriculum developers to provide insights into models for educational development to help ensure high quality learning for 2020 and beyond.

For many of the teaching staff in Universities quality assurance in higher education has a negative connotation, despite the underlying intention to improve the learning experience. It is our experience that talk about a quality assurance activity often elicits a deep sigh of resignation from the collegiate. Quality Assurance (QA), for many, is associated with long reports, gathering statistical data on throughput rates, mean time to graduation, documenting assessment methods and justifying that student performance is in accordance with the standards set out in the course and program goals.

This reaction to QA is problematic for several reasons, especially since the academy is a central stakeholder and has much to gain from the results of a relevant, tailored, QA approach. This paper provides no silver bullet with regard to changing this attitude, but does address some aspects of quality assurance and identifies aspects of QA systems that have the potential to act as a constructive and positive influence on educational practice.
Our work is based our personal experiences, both positive and negative, in being involved in different capacities in quality assurance efforts regarding degree programs, as well as literature studies. Earlier analysis of the literature related to quality in higher educational contexts identified three clearly identifiable perspectives [1]. Recent work on the development of competency frameworks for higher education in computing [2] emphasises a fourth, based on bodies of knowledge enshrined in model and reference curricula.

We examine the process from these four different perspectives, each founded on a view of the purpose of education. Since these perspective affect the focus and nature of how quality might be perceived each has an overarching influence on understanding the process and the outcomes. In the remainder of this paper we propose that education can be understood from four rather different perspectives, namely:

- Service - where identifying who is served, the customer, is crucial.

- Process - where the focus is on formative structures in the education.

- Body of Knowledge - where the focus is on the knowledge components in the education.

- Holistic personal development - where there is a focus on a professional identity.

These perspectives need not be seen as silos, since each perspective has aspects of the others embedded in it, or overlapping with it. However these perspectives can be seen to provide a focus for a QA process of processes. Any set of QA processes are, regardless of perspective, anchored in contexts, like national and institutional regulatory structures and political agendas. It is essential that these contextual aspects be both explicit and open to debate in the developing discourse surrounding higher education. Understanding is a key concept here, since it should be shared by all stakeholders. To have a common vocabulary to facilitate the discourse is thus essential. We will expand on these aspects of the QA process in order to set the scene for contemplations and suggestions regarding how the process could be enhanced toward a more positive participation among stakeholders, thus providing a better environment for the generation of constructive out- 
comes.

We use the EMV framework presented in [3], [4] to illustrate interdependence between stakeholders in the QA process. Identifying and understanding stakeholders and the relationship between them is a major factor in a constructive QA process. This model will provide a framework for illustrating and discussing this interdependence, where for instance similarities and differences between them is essential to capture. We will, furthermore, introduce a framework for describing (professional) competencies in order to provide a base for the particular discourse in the QA process. This framework, CoLeaF [2], can be used to clearly define outcomes of the QA process as well as capturing both components and processes in an educational setting. We argue that these frameworks will provide a setting that can be constructively used in QA processes at different levels, such international curricula standards, national or institutional degree evaluations, and course level developments. Our arguments are based on viewing the quality assurance process from the different perspectives and especially identifying the need to be able to share a common language (understanding) between stakeholders.

\section{Different PERspectives on the QuAlity ASSURANCE PROCESS}

Modelling the motivations and arguments for quality assurance requires the evaluator of the education to define what aspects of education are to be valued, and indeed how education itself can be conceived. In the work of Pears [1] three definitions are developed and argued for, education as service, process and expanding intellectual capacity of the individual. To these we add a fourth, education viewed as a Body of Knowledge.

\section{A. A Service}

Conceptualising education as a service has been a popular approach to QA in Australia and the United States, where Higher Education is offered on a personal payment model. In such a system it is tempting to view education as a commodity or service, and to evaluate quality in connection to expectations and experiences [5]. The view of education as a service gives a significant degree of agency to the student, who as the consumer, and purchaser of the service, becomes also central to defining key elements of what "qualities" of education are valued, and rewarded.

\section{B. A Process}

The process perspective focus on the organisation of a degree program, where quality lies in how well the organisation is set up and carried out. In its extreme it is independent of the students. This perspective is perhaps most clearly illustrated by the CDIO concept [6], [7]. CDIO is an engineering flavoured model of capturing education, where the idea is to model how courses in a degree program can be described as supporting Conceive, Design, Implement and Operate complex systems. The focus in CDIO is on creating a process that takes students in an organised manner from start to finish in their education.

\section{A Body of Knowledge Perspective}

The emphasis of this perspective is on the knowledge components needed in a degree program. This can typically be described in quite some detail and is something that can be evaluated against some clearly defined criteria. Especially the early ACM/IEEE curricula documents had content that match this view. This perspective is limited when taken by itself, since questions such as "how" and "why" are not relevant.

\section{Holistic Personal Development}

The holistic developmental perspective was central to the Humboltian model of education, and also argued by Dewey and colleagues. However, these discussions were part of a larger discourse on the challenges facing modern societies. Rapid industrialisation in Europe strongly influenced the development of pragmatic and utilitarian views of higher education by visionaries such as Humboldt, however the focus of his interest was largely in the development of the citizen [8]. John Dewey and his compatriots proposed that a central goal of higher education is "assisting individuals to develop the capacities to realise their vocation (i.e., to be effective in their preferred occupation)" [9]. In this context the role of higher education in a broader political sphere, the desire to understand the effects of education in a broader sense lead to a focus both on the nature of the educational endeavour and also the major stakeholders.

\section{Stakeholders In The Quality Assurance PROCESS}

Having a clear view of who the stakeholders are and the role they play in the context of a particular QA process is a key ingredient for being constructive. Who the stakeholders are depends on the perspective of the QA process. Moll [10] argues that an educational program should engage students, academics, employers, government agencies, and the wider society. We will add educational institutions and professional organisations to this list in our discussion. We will first present the EVM (Educational Value Model) framework as an example of how to capture and describe interrelations between stakeholder in a QA process, before expanding on the different stakeholders. The European Standards and Guidelines for quality assurance (ESG) model [11] developed by the European Association for Quality Assurance in Higher Education (ENQA) [12] is a relatively modern framework for QA, which we will relate to the presented stakeholders.

\section{A. The EVM Framework}

The Educational Value Model (EVM) as depicted in figure 1 has been used to investigate different aspects related to the interrelation between students, academics and higher education structures. The EVM framework provides a foundation where issues can be identified and studied by applying relevant theories. The investigation of student perspectives on the goal of STEM higher education addressed in [3] is quite relevant for issues in most QA processes. In that example the Reasoned Action Approach (RAA) developed by Fishbein 


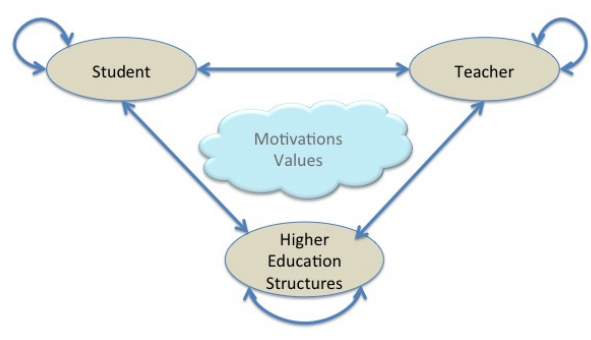

Fig. 1. Overview of the EVM Analytic Framework

and Ajzen [13] was brought in to the framework and used as an analysis tool. The interactions between the identified actors (stakeholders) were in that example captured in an intellectual alignment model. The investigation of the role of students' professional identity development addressed in [4] is another example of using the EVM framework and the RAA theory, where the arrows between the actors had a different interpretation better serving the issue to be described and investigated.

\section{B. Students}

Students have a rather special stakeholder position, in that they of course have a major stake in the education process in terms of the value for themselves. They can, however, also be viewed as a consumer, or even product of the education being evaluated. The former role is in line with the holistic personal development perspective, whereas the latter role is most clearly manifested in the service perspective, but also in the process and the body of knowledge perspectives.

\section{Academics}

Academics have different roles in the educational environment, for instance as teachers of courses, members of advisory boards, and directors of study programs. Analysis of systems and structures in this context can lend us insight into the educational perspectives that predominate. An examination of course descriptions and formal educational guidelines and recommendations provided by the Association for Computing Machinery [14] they tend to have on education is mainly the process and the body of knowledge perspectives.

\section{Educational Institutions}

The way education is perceived by educational institutions vary from quite pragmatic economic aspects to more altruistic educating for the greater good for the individual and/or society. Other quite pragmatic aspects is to tailor the education after the profile of its academics. Some of these aspects are not politically correct to state, but might still be quite a force behind their agenda. To look good on rankings of various kinds can also be a, perhaps not explicitly stated, goal for an educational institute.

\section{E. Employers}

In models of education that emphasise relevance and utility the status of the employer is often highly ranked. Employers are not a homogeneous group, small start-up companies have different needs and views compared to large multinational companies. There is for instance a difference between wanting graduates that can be productive from day one and wanting graduates that have a potential to learn and adapt in order to be valuable in the long term.

\section{F. Professional Organisations}

Professional organisations often have a clear goal of ensuring the quality of degrees. The professional competency of graduates is clearly on the agenda, but since this is difficult to access it is not uncommon for such organisations to have a focus on the body of knowledge perspective.

\section{G. Society}

Society places more systemic demands on education. In some philosophical positions society is seen as the ultimate beneficiary of education. Education equips citizens to participate in society to the fullest extent, and prepares them to make the high quality decisions needed to ensure continued, or enhanced, socio-economic success. In this sense education is an investment in the future, as well as the individual. However, shifting the focus of benefit to a broader societal plane also acts to elicit a discourse in education which emphasises maximising the ability of that society to leverage its human capital to achieve socio-economic goals.

\section{H. The ESG Model for Quality Assurance}

Many European countries base their QA processes on the European Standards and Guidelines for Quality Assurance (ESG) model. The current version of ESG was published in 2015 and is a modernisation of an earlier proposal (ESG 2005). The ESG is introduced as follows. "The ESG 2015 is based on four principles: that the primary responsibility lies with higher education institutions for the quality and quality assurance of their provision; that quality assurance needs to respond to the diversity of higher education systems, institutions, programs, and students; that quality assurance needs to support the creation of a quality culture; and that quality assurance takes into account the needs and expectations of students, other stakeholders, and the society." [15]

That is, ESG highlights both the types of stakeholders considered by the standard as well as the relations between them. The perhaps most surprising and, as we see it constructive, principle is the creation of a quality culture. We argue that there is a need to be concrete about what this means and to provide a vocabulary for a constructive discourse with enhanced quality in mind and recognising that quality is a relative aspect depending on which stakeholder perspective one adopts. 


\section{Professional) Competencies and the Quality ASSURANCE PROCESS}

The description of professional competencies can be used to capture central aspects in a QA process. Here we present a framework that can be used to capture many aspects of developing professional competencies in an educational concept, which in many ways can be seen as something quite important to catch in a QA process.

The development of professional competencies can be seen as a central ambition for any engineering degree program, and can thus serve as a common platform upon which to build QA processes. The concept of professional competency is intuitively linked to something someone in a given profession is supposed to be good at. There are several approaches to reaching more formal definitions of what a (professional) competency is but they share a common basic premise that competence is the integration of knowledge and skills, combined with a situational and contextual element which evinces a number of dispositional aspects of the learner. What in Aristotelian terms is ofter referred to as Phronesis or prudential judgement [16]. This structure has been influential in attempts to describe processes in higher education. One issue with much of the literature is a lack of standardised vocabulary available when discussing the concept. This is a problem for further investigation because the ambiguities of everyday language become transferred to the more technical terminology that is needed with academic investigation. In particular, the term "competency" and its cognates, competence, capability, and capacity have been given multiple definitions by different authors. We argue that the definition used in the CoLeaF framework [2] can serve as a good candidate for a joint vocabulary .

With regard to the definition of the term "competency", following Sandberg and Pinnington [17], we can draw out three broad domains in which of the word is used. The first of these is competency as a prerequisite. The word here refers to specific educational or training requirements necessary for permission to practice within a particular occupation. Secondly, we can see competency as a measurement of outcome, that is, performance to a set standard. Finally, we can view competency in terms of the exercise of a capability when accomplishing specific work tasks, that is, competency as a practical accomplishment [18]. It is this third description that the CoLeaF framework takes up for use in a university context.

With regard to the structure of a competency, there is a considerable degree of evidence that the triadic structure of knowledge, skills and dispositions is important to understanding the exhibition of proficiency within a learning environment. Moreover, it is necessary to understand this internal relationship in order to understand the integrative character of a competency, over and above it simply being a reductive aggregate of knowledge, skill and dispositional elements, which can be studied separately. There are aspects of the structure which require further explanation, such as the character of the various components and how they relate to each other. For example, if one considers the knowledge element associated with a competency, it is clear, e.g. from studies of work-based learning competencies, that there are a number of different types of knowledge which contribute to this component. Codified knowledge [19] is different from, say, episodic knowledge [20] and both differ from the type of implicit understanding of tasks which relies on tacit knowledge [21]. Alongside this categorisation of knowledge, we can also see a taxonomy of skills, e.g. the proposal of the National Research Council in 2012 [22]. So far, the epistemic and skill components have been treated in an undifferentiated way, although a more comprehensive understanding of the concept of competency would require a more nuanced approach.

Further clarification of the dispositional aspect, which is generally considered vital for the exercise of a competency, is also required, since this draws on a philosophical position distinct to the knowledge and skill components. To understand this in more detail we need to return to the epistemelogical underpinnings of our understanding of the application of knowledge in context.

While important, internal structure is not the only way of conceptualising competency. It is also possible to characterise the way in which different competencies differ from each other with regard to their individual characteristics. Mulder [9] identified ten "dimensions", that is, more or less independent ways in which a competence can be characterised. These are:

- Centrality: the degree to which a competency is central to a professional engaged in some field. The range would be from central to peripheral. Central competencies are essential for effective performance and being used frequently, whereas peripheral competencies are less important. So, for example, if we consider the competencies of a software engineer, a programming competency might be considered central whereas a financial management competency might be considered more peripheral.

- Contextuality: the degree to which a particular competency is generic or context specific. Here, contextspecific means being more-or-less related to a specific situation which may be an individual circumstance or a particular content domain. More generic competencies are applicable across wider contexts. Social competencies tend to be generic whereas those derived from educational contexts tend to be more specific. There is a considerable consensus though that competencies only get concrete meaning when applied in a specific situation [Bartram, D. et al., 2008].

- Definability: the degree to which a specific competency can be clearly defined.

- Developability: the degree to which a competency can be developed, in the sense of growth mindset [23], or is seen as fixed by personal cognitive qualities.

- Dynamic nature: the degree to which a competency is triggered by or expressed in certain circumstances. Some competencies, such as empathy, appear to be part of the basic human condition, whereas others, such as programming competency, come into action in specific 
circumstances.

- Knowledge inclusion: the degree to which knowledge is considered to be important. Some competencies, especially those drawn from a practical or vocational context, appear to have a relatively minor knowledge component, being mostly dependent on the skill of the practitioner; in other competencies, the knowledge component is significant. An example of the former would be the competency to make a wooden cabinet carpentry joint which relies on the skill of the carpenter. An example of the latter might be a competency to solve a differential equation which does have a large knowledge component.

- Measurability: the degree to which competencies can be measured. Some competencies appear to be directly measurable, such as the competency of singing a particular note. Others appear harder to measure because they involve assessing various proxies which may require significant interpretation or analysis.

- Mastery level: the level to which a competency is achieved. Some competencies, such as driving a car, can be more-or-less fully achieved after a period of study. Others, such as the competency for drawing a portrait, appear to be more open-ended.

- Performativity: the degree to which a competency relates to performance. This may be linked to measurability if the competency is based on an explicit demonstration but there are some competencies which are less easy to measure but are nevertheless essentially performative, e.g. intercultural competency.

- Transferability: the degree to which competencies can be acquired in one domain and then successfully applied in other professional situations.

The importance of this list is, not necessarily to claim an exhaustive taxonomy of properties that characterise competency but rather to illustrate the complex nature of the concept and the various ways that it can provide insight into the educational context of the learner. Both the structural viewpoint, and the dimensional perspective that describes the functional aspects of competencies as they relate to external observers and assessors, demonstrate that the concept has a rich and wide area of application. These are arguments for why professional competencies can be a useful focus for discussing different aspects of the QA process.

\section{Discussion}

The principle that quality assurance needs to support the creation of a quality culture stated in ESG [11] is close to the heart of what we mean by being constructive in a QA process. This is however not easy to achieve, not least to the increase demands to be productive. This obstacle has different aspects, one being that there is less time to do other things than those clearly associated with a position, such as teaching and doing research for academics, and another being a sense of being controlled and measured according to some out-ofcontrol variables. It is thus of high importance that there needs to be ample time set aside for the QA process and also that the intentions behind the process are clearly articulated.

We opine that particular attention should be paid to more holistic approaches to QA, which integrate a wider range of factors that have a direct impact on the reputation and holistic quality of academic institutions. In particular the work of Owlia and Aspinwall [24], [25] is relevant to a more nuanced discussion of what constitutes quality in an academic setting.

The integration of these types of broader indicators, implies that a more systemic view of higher education organisations and their broader mission is desirable. Commoditisation of higher education [26], particularly with a focus on graduate competencies, or graduate attributes has both positive and negative impact on how teachers at universities engage with their teaching duties. These types of issues need to be broadly discussed and situated in the local educational culture if a more widely accepted approach to QA integral, and symbiotic with, staff culture and teaching values is to be achieved.

\section{CONCLUSION}

We observe that how quality is defined, measured, improved and assured, depends on how education is conceived and modelled. Highly valued attributes of services as measured by the gap between experience and expectations, while relevant to student satisfaction, are not necessarily good indicators of a high-performing educational environment. As Roger Säljö the educationalist observed [27]

"Learning is not fun! Learning involves stress, and pushing yourself beyond your comfort zone. If you are completely relaxed and having fun you are probably not learning anything. Learning hurts!"

Broader definitions of quality are necessary. These definitions should also be supported by an embedded culture of quality within the institution, and that in turn supported by the development of embedded and intrinsic Key Performance Indicators (KPI's), which provide forward looking input to a Total Quality Management process, rather than the current retrospective QA approach.

Consequently, this paper has paper focused on one of the guiding ESG principles, namely, that QA processes should foster a quality culture, which we view as an essential component in any constructive QA approach. Taking an academic perspective, we propose to refocus QA activities using the concept of professional competencies as a theme. This provides a new framing, within which the higher education profession can start to discuss what a QA process contributes in a broader academic context. We also suggest that the CoLeaF framework provides a valuable and potent setting for deriving a common vocabulary between stakeholders. Finally the EVM framework is presented as a tool with which to understand and reason about different interrelations between the stakeholders, where having a clear perspective on what is to be achieved with the QA process can more easily be articulated. 


\section{REFERENCES}

[1] A. Pears, "Does Quality Assurance Enhance the Quality of Computing Education?" in Proceedings of the 12th Australasian Computer Science Education Conference, CRPIT, T. Clear and J. Hamer, Eds., vol. 103. ACM Press New York, NY, USA, 2010.

[2] S. Frezza, A. Pears, M. Daniels, V. Kann, A. Kapoor, R. McDermott, A.-K. Peters, C. Wallace, M. Sabin, and \. Cajander, "Modeling global competencies for computing education," in Proceedings of the 23rd Annual ACM Conference on Innovation and Technology in Computer Science Education. ACM, 2018, pp. 348-349.

[3] A. Nylén, Å. Cajander, M. Daniels, A. Pears, and R. McDermott, "Why are we here? : Student perspectives on the goal of higher education," in Proc. 47th ASEE/IEEE Frontiers in Education Conference, 2017.

[4] A. Nylén, M. Daniels, A. Pears, Å. Cajander, R. McDermott, and V. Isomöttönen, "Why are we here? The Educational Value Model (EVM) as a framework to investigate the role of students' professional identity development," in Proc. 2018 IEEE Frontiers in Education Conference (FIE), Oct 2018.

[5] F. Abdullah, "Measuring service quality in higher education: HEdPERF versus SERVPERF," Marketing Intelligence \& Planning, vol. 24, no. 1, pp. 31-47, 2006.

[6] K.-F. Berggren, D. Brodeur, E. F. Crawley, I. Ingemarsson, W. T. G. Litant, J. Malmqvist, and S. Östlund, "CDIO: An international initiative for reforming engineering education," vol. 2, no. 1, p. 4, 2003.

[7] D. R. Brodeur and E. F. Crawley, "CDIO and Quality Assurance: Using the Standards for Continuous Program Improvement," Engineering Education Quality Assurance, pp. 211-222, 2009. [Online]. Available: http://dx.doi.org/10.1007/978-1-4419-0555-0_17

[8] M. G. Ash, "From 'Humboldt' to 'Bologna': history as discourse in higher education reform debates in German-speaking Europe," Education and the Knowledge-Based Economy in Europe, pp. 4161, Jan. 2008. [Online]. Available: https://brill.com/view/book/edcoll/ 9789087906245/BP000004.xml

[9] M. Mulder, "Competence Theory and Research: A Synthesis," in Competence-based Vocational and Professional Education, ser. Technical and Vocational Education and Training: Issues, Concerns and Prospects. Springer, Cham, 2017, pp. 1071-1106. [Online]. Available: https://link.springer.com/chapter/10.1007/978-3-319-41713-4_50

[10] I. Moll, "Curriculum responsiveness: The anatomy of a concept." in Curriculum responsiveness: Case studies in higher education. South African Universities Vice-Chancellors Association, Nov. 2004, pp. 1-9.

[11] European Association for Quality Assurance in Higher Education (ENQA), Standards and guidelines for quality assurance in the European Higher Education Area (ESG). ENQA Brussels, 2015.

[12] ENQA, "European association for quality assurance in higher education (ENQA)," https://enqa.eu/, 2013.
[13] M. Fishbein and I. Ajzen, Predicting and changing behavior: The reasoned action approach. Taylor \& Francis, 2011.

[14] ACM, "ACM Curricula Recommendations," Tech. Rep. [Online]. Available: https://www.acm.org/education/curricula-recommendations

[15] E. A. for Quality Assurance in Higher Education, Standards and guidelines for quality assurance in the European Higher Education Area (ESG), 2015. [Online]. Available: http://www.enqa.eu/index.php/ home/esg/

[16] J. Noel, "On the Varieties of Phronesis," Educational Philosophy and Theory, vol. 31, no. 3, pp. 273-289, Jan. 1999. [Online]. Available: https: //www.tandfonline.com/doi/full/10.1111/j.1469-5812.1999.tb00466.x

[17] J. Sandberg and A. H. Pinnington, "Professional Competence as Ways of Being: An Existential Ontological Perspective," Journal of Management Studies, vol. 46, no. 7, pp. 1138-1170, Nov. 2009. [Online]. Available: https://onlinelibrary.wiley.com/doi/abs/10. 1111/j.1467-6486.2009.00845.x

[18] S. Gherardi, Practice-based theorizing on learning and knowing in organizations. Sage Publications Sage CA: Thousand Oaks, CA, 2000.

[19] M. Eraut, "Knowledge, Working Practices, and Learning," in Learning Through Practice: Models, Traditions, Orientations and Approaches, S. Billett, Ed. Dordrecht: Springer Netherlands, 2010, pp. 37-58. [Online]. Available: https://doi.org/10.1007/978-90-481-3939-2_3

[20] C. Bereiter, "Design research for sustained innovation," Cognitive Studies, vol. 9, no. 3, pp. 321-327, 2002.

[21] P. Duguid, "“The Art of Knowing": Social and Tacit Dimensions of Knowledge and the Limits of the Community of Practice," The Information Society, vol. 21, no. 2, pp. 109-118, Apr. 2005. [Online]. Available: https://doi.org/10.1080/01972240590925311

[22] J. W. Pellegrino and M. L. Hilton, Eds., Education for Life and Work: Developing Transferable Knowledge and Skills in the 21st Century. Washington, D.C.: National Academies Press, Dec. 2012. [Online]. Available: http://www.nap.edu/catalog/13398

[23] C. S. Dweck, Self-Theories: Their Role in Motivation, Personality, and Development. Philadelphia: The Psychology Press, 1999.

[24] M. S. Owlia and E. M. Aspinwall, "A framework for the dimensions of quality in higher education," Quality Assurance in Education, vol. 4, no. 2, pp. 12-20, 1996.

[25] — " "TQM in Higher Education - A Review," International Journal of Quality \& Reliability Management, vol. 14, no. 5, pp. 527-543, 1997.

[26] E. Pikkarainen, "Competence as a Key Concept of Educational Theory: A Semiotic Point of View." Journal of Philosophy of Education, vol. 48, no. 4, pp. 621 - 636, 2014. [Online]. Available: https://search-ebscohost-com.ezproxy.its.uu.se/ login.aspx ?direct=true $\& \mathrm{db}=$ eue $\& \mathrm{AN}=99596582 \&$ site=ehost-live

[27] R. Säljö, "The nature of learning." 2009, personal communication. 